\title{
Nonlinear coupled CNN models for multiscale image analysis
}

\author{
F. Corinto, M. Biey and M. Gilli \\ Department of Electronics, Politecnico di Torino \\ Corso Duca degli Abruzzi, 24 \\ I-10129 Torino, Italy
}

\begin{abstract}
A CNN model of partial differential equations (PDEs) for image multiscale analysis is proposed. The model is based on a polynomial representation of the diffusivity function and defines a paradigm of polynomial CNNs, for approximating a large class of nonlinear isotropic and/or anisotropic PDEs. The global dynamics of spacediscrete polynomial CNN models is analyzed and compared with the dynamic behavior of the corresponding space-continuous PDE models. It is shown that in the isotropic case the two models are not topologically equivalent: in particular discrete CNN models allow one to obtain the output image without stopping the image evolution after a given time (scale). This property represents an advantage with respect to continuous PDE models and could simplify some image preprocessing algorithms.
\end{abstract}

\section{Index Terms}

\section{INTRODUCTION}

Partial Differential Equation (PDE) based models have shown to be useful for image preprocessing (multiscale analysis) [1], [2], [3], [4]. They can be classified in three main categories: a) linear isotropic filters; b) nonlinear isotropic filters; c) nonlinear anisotropic filters. Linear isotropic filtering (heat equation) was proved to be the only linear model that satisfies all the multiscale analysis requirements [2]. However it presents the disadvantage of blurring important features in image processing, like edges. Nonlinear isotropic filters (in particular the Perona-Malik filter [5]) were introduced for overcoming the limitations of linear models and are based on the idea of adapting the diffusivity to the gradient of the evolving image. They do not exhibit good performances in images containing noisy edges and coherent flow-like structures. In such cases they can be replaced by anisotropic models [1], that have found significant applications in medical imaging and computer-aided quality control [1]. The main drawback of PDE based techniques is that from a computational point of view they are rather time-consuming and therefore they are not suitable for real-time image processing.

In parallel with the mathematical studies on the application of nonlinear PDEs to image processing, in the electrical engineering community a new paradigm of neural network, called cellular neural network (CNN) ([6], [7], [8], [9]), was introduced and shown to be able to solve several complex computational problems.

As far as the applications are concerned, the implementation of PDE-based algorithms onto CNNs allows one to afford real-time image processing [10]. From a theoretical point of view, the relationship between PDE and CNN dynamics has been investigated in several papers. In [11] it was shown that, in general, CNN dynamics represents a broader class than PDEs.

In this manuscript a CNN model of partial differential equations for image multiscale analysis is proposed. The model is based on a polynomial representation of the diffusivity function and defines a paradigm of polynomial CNNs, which approximates a large class of nonlinear isotropic and anisotropic PDEs, and also some non-PDE models [12]. The global dynamics of space-discrete polynomial CNN models is analyzed and compared with the dynamic behavior of the corresponding space-continuous PDE 
models. It is shown that in the isotropic case the two models are not topologically equivalent: in particular discrete CNN models allow one to obtain the output image without stopping the image evolution after a given time (scale). This property represents an advantage with respect to continuous PDE models and could simplify some image preprocessing algorithms.

\section{PDE BASED CNN MODELS}

According to [1], [2], a gray-level image is defined as a real bounded function $u_{0}(x, y): \boldsymbol{R}^{2} \rightarrow \boldsymbol{R}$, where $u_{0}(x, y)$ denotes the gray level value at the point $(x, y)$. Introducing an artificial time $t$, an image $u(t, x, y)$ is assumed to evolve, according to a PDE of the form:

$$
\begin{array}{r}
\frac{\partial u(t, x, y)}{\partial t}=\boldsymbol{F}[u(t, x, y)] \\
u(0, x, y)=u_{0}(x, y)
\end{array}
$$

where $\boldsymbol{F}: \boldsymbol{R} \rightarrow \boldsymbol{R}$ is an operator that typically depends on the image and on its first and second order space derivatives and characterizes the given algorithm; $u_{0}(x, y)$ represents the initial condition. Hereinafter we assume that each image $u(t, x, y)$ exhibits Neumann type space boundary conditions.

The solution of equation (1), denoted by $T_{t}\left(u_{0}(x, y)\right)=u(t, x, y)$, represents the processed image at the scale (time) $t$. As shown in [2], the sequence of images $u(t, x, y)$, that is the images generated from $u_{0}(x, y)$ by the operator $T_{t}(\cdot)$, performs a multiscale analysis satisfying some selected basic principles (called axioms in [2]).

\section{A. Isotropic diffusion based CNN models}

It is well known that the simplest multiscale analysis is obtained by using linear isotropic diffusion, i.e. by exploiting the following PDE (heat equation):

$$
\frac{\partial u}{\partial t}=\operatorname{div}[\nabla u]=\frac{\partial^{2} u}{\partial x^{2}}+\frac{\partial^{2} u}{\partial y^{2}}
$$

To overcome the drawbacks of linear diffusion, Perona and Malik [5] proposed the following PDE describing a nonlinear isotropic diffusion:

$$
\frac{\partial u}{\partial t}=\operatorname{div}\left[g\left(\|\nabla u\|^{2}\right) \nabla u\right]
$$

where the original image $u(0, x, y)=u_{0}(x, y)$ is used as initial condition, $\nabla u$ and $\|\nabla u\|$ denote the image gradient and its magnitude respectively and $g(\cdot)$ represents the diffusivity function. The following diffusivity functions were proposed by Perona and Malik [5]:

$$
g\left(\|\nabla u\|^{2}\right)=e^{-\left(\frac{\|\nabla u\|}{k}\right)^{2}}, \quad g\left(\|\nabla u\|^{2}\right)=\left[1+\left(\frac{\|\nabla u\|}{k}\right)^{2}\right]^{-1}
$$

where $k$ is a scalar parameter.

Finite difference approximations of Perona-Malik equation (3) have been investigated in several papers ([1], [13], and [14]).

Let us suppose that the continuous space domain is composed by $N \times M$ points arranged on a regular grid and let us denote the position of a point with two indexes $(i, j) ; u_{i j}$ represents the pixel value, obtained by sampling the image $u(t, x, y)$ at the point $\left(x_{i}, y_{j}\right)=(h i, h j)$, where $h$ is the grid size. It turns out that among the many approaches for solving PDEs, the finite difference method is the most suited to obtain a system of coupled ordinary differential equations, that can be mapped onto a CNN structure. 


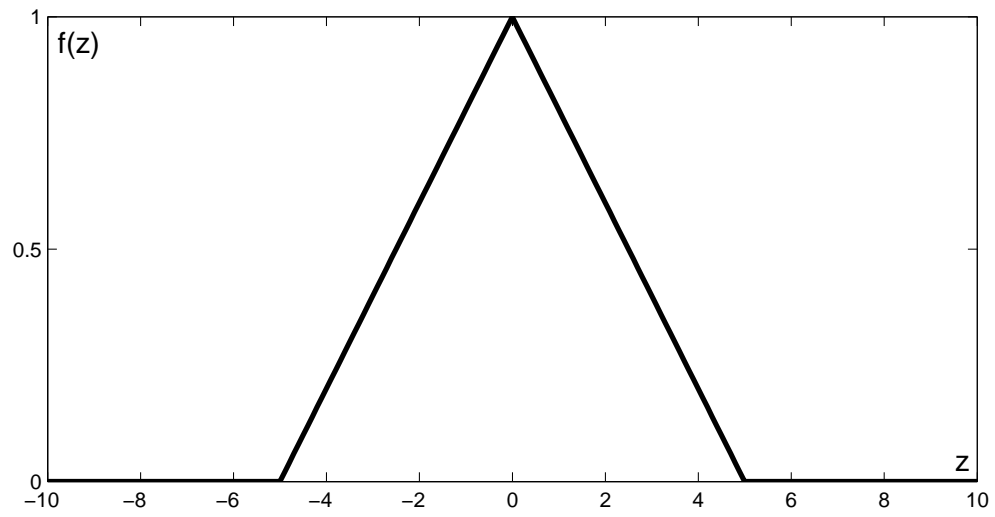

Fig. 1. Basis function $f(z)$ for $m=5$.

By using a finite difference approximation, a consistent semidiscrete version of the Perona-Malik model (3) can be written as $[1](1 \leq i \leq N$ and $1 \leq j \leq M)$ :

$$
\frac{d u_{i j}(t)}{d t}=\sum_{(k l) \in N_{i j}} \frac{g_{k l}+g_{i j}}{2 h^{2}}\left(u_{k l}-u_{i j}\right)
$$

where $N_{i j}=\{(i-1, j),(i+1, j),(i, j-1),(i, j+1)\}$ and $g_{k l}$ and $g_{i j}$ are evaluated by approximating the image gradient through the central difference around the points $\left(x_{k}, y_{l}\right)$ and $\left(x_{i}, y_{j}\right)$ respectively. As an example, $g_{i j}=g\left(\left\|\widetilde{\nabla} u_{i, j}\right\|^{2}\right)$ with

$$
\left\|\widetilde{\nabla} u_{i, j}\right\|^{2}=\left(\frac{u_{i+1, j}-u_{i-1, j}}{2 h}\right)^{2}+\left(\frac{u_{i, j+1}-u_{i, j-1}}{2 h}\right)^{2} .
$$

In order to implement (5) in a general feasible polynomial CNN architecture, let us consider the following basis function (see Fig. 1):

$$
f(z)=1-\left|\frac{1}{2}\left(\left|\frac{z}{m}+1\right|-\left|\frac{z}{m}-1\right|\right)\right|
$$

and let us approximate the $g(\cdot)$ functions (4) with the following expression:

$$
g(z) \cong \gamma(z)=\sum_{p=1}^{Q} c_{p} f^{p}(z)
$$

It is worth observing that $f(z)$ is different from zero only on the finite domain $z \in(-m, m)$; this allows us to control the nonlinear diffusion process according to the threshold $m$. Hence, this property is also valid for $\gamma(z)$.

The parameter $m$ depends on the admissible error $\widehat{e}$ between $g(\cdot)$ and $\gamma(\cdot)$ at the point $z=m$. This allows us to rewrite $m$ as $m=k^{2} \delta(\widehat{e})$, where $\delta(\widehat{e})=-\ln (\widehat{e})$ or $\delta(\widehat{e})=\left((\widehat{e})^{-1}-1\right)$ for the functions reported in (4).

The parameters $c_{1}, \ldots, c_{Q}, Q$ and $m$ are chosen in such a way that $\gamma(z)$ interpolates $g(z)$ in a set of $Q$ uniform spaced points within the interval $[-m, m]$. It follows that $\gamma(z)$ and $g(z)$ exhibit the same properties, i.e. $\gamma(z)$ is constrained to be a positive and bounded function $(0 \leq \gamma(z) \leq 1)$; these conditions ensure that:

$$
g_{i j} \cong \gamma_{i j}=\sum_{p=1}^{Q} c_{p} f^{p}\left(\left\|\widetilde{\nabla} u_{i, j}\right\|^{2}\right)
$$


By replacing $g_{i j}$ and $g_{k l}$ in (5) with the approximate expressions $\gamma_{i j}$ and $\gamma_{k l}$, we obtain a general, nonlinear PDE based, polynomial CNN model (PCNNs):

$$
\frac{d u_{i j}(t)}{d t}=\sum_{(k l) \in N_{i j}} \Gamma_{k l}\left(u_{k l}-u_{i j}\right)
$$

where $\Gamma_{k l}$ is reported below

$$
\Gamma_{k l}=\sum_{p=1}^{Q} \frac{c_{p}}{2 h^{2}}\left[f^{p}\left(\left\|\widetilde{\nabla} u_{k, l}\right\|^{2}\right)+f^{p}\left(\left\|\widetilde{\nabla} u_{i, j}\right\|^{2}\right)\right] .
$$

and defines the following nonlinear template, whose structure is identical for each processing cell:

$$
\left[\begin{array}{ccc}
0 & \Gamma_{i-1, j} & 0 \\
\Gamma_{i, j-1} & 0 & \Gamma_{i, j+1} \\
0 & \Gamma_{i+1, j} & 0
\end{array}\right]
$$

\section{B. Anisotropic diffusion based CNN models}

In nonlinear isotropic filters, the diffusion process only depends on the gradient magnitude. In certain applications [1] better performances are achieved if the diffusivity is not identical in all the directions. This requirement can be fulfilled, by introducing a suitable diffusion matrix, that changes the orientation of the gradient.

In [1] the following anisotropic PDE equation was proposed:

$$
\frac{\partial u}{\partial t}=\operatorname{div}[\boldsymbol{D} \nabla u]
$$

where $\boldsymbol{D}$ is a positive definite $2 \times 2$ matrix, depending on the gradient. It is easily derived that linear and nonlinear isotropic diffusion can be obtained with $\boldsymbol{D}=\boldsymbol{I}$ and $\boldsymbol{D}=g\left(\|\nabla u\|^{2}\right) \boldsymbol{I}$ respectively (where $\boldsymbol{I}$ denotes the identity matrix).

Usually, the matrix $\boldsymbol{D} \in \boldsymbol{R}^{2 \times 2}$ is chosen to be a function of $\nabla u$ (see [1]):

$$
\boldsymbol{D}=\boldsymbol{S} \boldsymbol{\Lambda}(\|\nabla u\|) \boldsymbol{S}^{T}
$$

where $\boldsymbol{\Lambda}(\|\nabla u\|)=\operatorname{diag}\left(\lambda_{1}(\|\nabla u\|), \lambda_{2}(\|\nabla u\|)\right)$ is a diagonal matrix containing the eigenvalues $\lambda_{1}, \lambda_{2}$ and $\boldsymbol{S}$ is a $2 \times 2$ matrix whose rows are the two corresponding eigenvectors $\boldsymbol{v}_{1}, \boldsymbol{v}_{2}$.

The main drawback of isotropic models (like Perona-Malik's) is that they might be not robust in presence of noise. In order to remove the noise at the edges it is necessary to rotate the flux according to the edge direction, in order to allow the diffusion along the edges and to prevent it perpendicularly. This can be obtained by choosing the matrices $S$ and $\boldsymbol{\Lambda}$ reported below:

$$
\boldsymbol{S}=\left(\begin{array}{cc}
\frac{\partial u}{\partial x} & \frac{\partial u}{\partial y} \\
\frac{\partial u}{\partial y} & -\frac{\partial u}{\partial x}
\end{array}\right) \quad \boldsymbol{\Lambda}=\left(\begin{array}{cc}
g\left(\|\nabla u\|^{2}\right) & 0 \\
0 & 1
\end{array}\right)
$$

that give rise to the following matrix $D$

$$
\boldsymbol{D}=\left(\begin{array}{ll}
a(x, y) & b(x, y) \\
b(x, y) & c(x, y)
\end{array}\right)
$$




$$
\begin{aligned}
& a(x, y)=g\left(\|\nabla u\|^{2}\right)\left(\frac{\partial u}{\partial x}\right)^{2}+\left(\frac{\partial u}{\partial y}\right)^{2} \\
& b(x, y)=\left[g\left(\|\nabla u\|^{2}\right)-1\right] \frac{\partial u}{\partial x} \frac{\partial u}{\partial y} \\
& c(x, y)=g\left(\|\left.\nabla u\right|^{2}\right)\left(\frac{\partial u}{\partial y}\right)^{2}+\left(\frac{\partial u}{\partial x}\right)^{2} .
\end{aligned}
$$

A standard space discretization scheme for equation (13), when the matrix $\boldsymbol{D}$ shown in (16) is exploited, allows one to obtain the following semi-discrete model:

$$
\frac{d u_{i j}(t)}{d t}=\sum_{(k l) \in \widehat{N}_{i j}} \Gamma_{k l}\left(u_{k l}-u_{i j}\right)
$$

where, the parameters $\Gamma_{k l}$ (with $(k l) \in \widehat{N}_{i j}=\{(k, l) \neq(i, j):|k-i| \leq 1 \bigwedge|l-j| \leq 1\}$ ) define the following nonlinear template, whose structure is identical for each processing cell $(i, j)$ :

$$
\left[\begin{array}{ccc}
b_{i-1, j}+b_{i, j-1} & a_{i-1, j}+a_{i, j} & -\left(b_{i-1, j}+b_{i, j+1}\right) \\
c_{i, j-1}+c_{i, j} & 0 & c_{i, j+1}+c_{i, j} \\
-\left(b_{i+1, j}+b_{i, j-1}\right) & a_{i+1, j}+a_{i, j} & b_{i+1, j}+b_{i, j+1}
\end{array}\right]
$$

In the above expression $a_{i, j}, b_{i, j}$ and $c_{i, j}$, represent the polynomial approximation of the functions $a(x, y), b(x, y)$ and $c(x, y)$ respectively, according to the following expressions:

$$
\begin{aligned}
& a_{i, j}=\gamma_{i, j}\left(\frac{u_{i+1, j}-u_{i-1, j}}{2 h}\right)^{2}+\left(\frac{u_{i, j+1}-u_{i, j-1}}{2 h}\right)^{2} \\
& b_{i, j}=\left(\gamma_{i, j}-1\right)\left(\frac{u_{i+1, j}-u_{i-1, j}}{2 h}\right)\left(\frac{u_{i, j+1}-u_{i, j-1}}{2 h}\right) \\
& c_{i, j}=\gamma_{i, j}\left(\frac{u_{i, j+1}-u_{i, j-1}}{2 h}\right)^{2}+\left(\frac{u_{i+1, j}-u_{i-1, j}}{2 h}\right)^{2} .
\end{aligned}
$$

where $\gamma_{i, j}$ is given by (9).

\section{Comparison Between Perona-Malik And POlynomial CNN MOdels}

Dynamical properties of Perona-Malik based filters (5) are summarized in [1] and [14]. It is well-known that system (5) converges toward a constant steady state solution, representing the average value of the initial image, because $g_{i j}$ and $g_{k l}$ are always different from zero. In order to obtain a non trivial output image, the system evolution has to be stopped after a finite time (usually called scale). In the most general case, the scale depends on the objects and on the characteristics of the input image, and hence there is no a priori known time for stopping image processing.

This problem can be overcome, by approximating the functions $g(\cdot)$, reported in equation (4)), (that are different from zero in the whole domain) with the expression $\gamma(\cdot)$ given by (8), that is different from zero for $|z|=\left\|\widetilde{\nabla} u_{i, j}\right\|^{2}<m$ (see Section II). This presents the advantage of stopping the evolution of the image when the approximated gradient magnitude $\left\|\widetilde{\nabla} u_{i, j}\right\|^{2}$ (for all $(i, j)$ ) is greater than the threshold $m$. As consequence, the output image exhibits a segmented structure.

The above behavior is possible because the set of equilibrium points of a PCNN system (10) is larger than that of the discretized Perona-Malik model. Such equilibrium points should satisfy at least one of the following two conditions:

1) $u_{i j}=u_{k l}$ for all $(k, l) \in N_{i j}$ and for all $(i, j)$ 
2) $\Gamma_{k l}=0$ for all $(k, l) \in N_{i j}$ and for all $(i, j)$

Condition 1 identifies those equilibrium points, that also the discretized Perona-Malik model possesses. Condition 2 identifies the set of equilibrium points that the discretized Perona-Malik model does not exhibit. It is derived that Condition 2 is equivalent to the constraints given by the following Proposition:

Proposition 1 : The condition $\Gamma_{k l}=0$ for all $(k, l) \in N_{i j}$ and for all $(i, j)$ is satisfied if and only if:

$$
\begin{array}{ll}
\left\|\widetilde{\nabla} u_{i, j}\right\|^{2} \geq m & \forall(i, j) \\
\left\|\widetilde{\nabla} u_{k, l}\right\|^{2} \geq m & \forall(i, j) \text { and } \forall(k, l) \in N_{i j} .
\end{array}
$$

Proof: By noting that the coefficient $\Gamma_{k l}$, shown in equation (11), is written as sum of nonnegative terms, it can be easily obtained that $\Gamma_{k l}=0$ for all $(k, l) \in N_{i j}$ and for all $(i, j)$ if and only if:

$$
\begin{aligned}
& f^{p}\left(\left\|\widetilde{\nabla} u_{i, j}\right\|^{2}\right)=0 \quad \forall(i, j) \\
& f^{p}\left(\left\|\widetilde{\nabla} u_{k, l}\right\|^{2}\right)=0 \quad \forall(i, j) \text { and } \forall(k, l) \in N_{i j} .
\end{aligned}
$$

The conditions above are verified if and only if the argument of the function $f(\cdot)$ (see equation (7)) is greater than $m$, that is:

$$
\begin{array}{ll}
\left\|\widetilde{\nabla} u_{i, j}\right\|^{2} \geq m & \forall(i, j) \\
\left\|\widetilde{\nabla} u_{k, l}\right\|^{2} \geq m & \forall(i, j) \text { and } \forall(k, l) \in N_{i j} .
\end{array}
$$

Note that if $m$ is chosen in such a way that only one of the above constraints (19), (20) is verified, then $\Gamma_{k l}$ is always different from zero. This implies that both systems converge toward the only equilibrium point represented by the average value of the input image.

In order to show the influence of $m$ on PCNN dynamics, we consider the very simple case of onedimensional images composed by $\mathrm{N}$ pixels (denoted by $u_{i}, 1 \leq i \leq N$ ). We denote with $i=\bar{\imath}$ a generic index and define the average value of the left and of the right side of the input image $u_{i}^{0}$ (with respect to index $\bar{\imath}$ ) as follows:

$$
\left\langle u_{l(\bar{\imath})}\right\rangle=\frac{1}{\bar{\imath}} \sum_{i=1}^{\bar{\imath}} u_{i}^{0}, \quad\left\langle u_{r(\bar{\imath})}\right\rangle=\frac{1}{N-\bar{\imath}} \sum_{i=\bar{\imath}+1}^{N} u_{i}^{0}
$$

The following Proposition holds:

Proposition 2 : Let us consider an input image such that:

(a) $u_{1}^{0}=\ldots=u_{\bar{\imath}-1}^{0}=u_{\bar{\imath}}^{0}=\left\langle u_{l(\bar{\imath})}\right\rangle$

(b) $u_{\bar{\imath}+1}^{0}=u_{\bar{\imath}+2}^{0}=\ldots=u_{N}^{0}=\left\langle u_{r(\bar{\imath})}\right\rangle$

If $\left|\left\langle u_{l(\bar{\imath})}\right\rangle-\left\langle u_{r(\bar{\imath})}\right\rangle\right| \geq 2 h \sqrt{m}$, then the input image represents an equilibrium point for the PCNN system defined by (10). If $\left|\left\langle u_{l(\bar{l})}\right\rangle-\left\langle u_{r(\bar{\imath})}\right\rangle\right|<2 h \sqrt{m}$, then the input image converges toward an equilibrium point corresponding to its average value.

Proof: In case of one-dimensional images, PCNN system (10) becomes:

$$
\dot{u}_{i}=\Gamma_{i-1}\left(u_{i-1}-u_{i}\right)+\Gamma_{i+1}\left(u_{i+1}-u_{i}\right)
$$


Owing to input image conditions, it is derived that only the following derivatives might be different from zero at the initial instant $t=0$ :

$$
\begin{aligned}
\left(\dot{u}_{\bar{\imath}}\right)_{\mid t=0} & =\Gamma_{\bar{\imath}+1}^{0}\left(u_{\bar{\imath}+1}^{0}-u_{\bar{\imath}}^{0}\right) \\
\left(\dot{u}_{\bar{\imath}+1}\right)_{\mid t=0} & =\Gamma_{\bar{\imath}}^{0}\left(u_{\bar{\imath}}^{0}-u_{\bar{\imath}+1}^{0}\right)
\end{aligned}
$$

where the coefficients, defined in (11), $\Gamma_{\bar{\imath}}^{0}=\gamma_{\bar{\imath}}^{0}+\gamma_{\bar{\imath}+1}^{0}$ and $\Gamma_{\bar{\imath}+1}^{0}=\gamma_{\bar{\imath}+1}^{0}+\gamma_{\bar{\imath}}^{0}$ are identical.

The necessary and sufficient conditions in order that $\left(\dot{u}_{\bar{\imath}}\right)_{\mid t=0}$ and $\left(\dot{u}_{\bar{\imath}+1}\right)_{\mid t=0}$ be null is that both $\gamma_{\bar{\imath}}^{0}$ and $\gamma_{\bar{\imath}+1}^{0}$ be zero, that is:

$$
\begin{aligned}
\gamma_{\bar{\imath}}^{0}=0 & \Longleftrightarrow\left|u_{\bar{\imath}-1}^{0}-u_{\bar{\imath}+1}^{0}\right| \geq 2 h \sqrt{m} \\
\gamma_{\bar{\imath}+1}^{0}=0 & \Longleftrightarrow\left|u_{\bar{\imath}}^{0}-u_{\bar{\imath}+2}^{0}\right| \geq 2 h \sqrt{m}
\end{aligned}
$$

It turns out that if the above conditions (28) are satisfied, then the input image $u_{i}^{0}$ is an equilibrium point. Since conditions (28) are equivalent to $\left|\left\langle u_{l(\bar{l})}\right\rangle-\left\langle u_{r(\bar{\imath})}\right\rangle\right| \geq 2 h \sqrt{m}$, this proves the first part of the Proposition.

If $\left|\left\langle u_{l(\overline{)})}\right\rangle-\left\langle u_{r(\bar{\imath})}\right\rangle\right|<2 h \sqrt{m}$, then (26) and (27) are different from zero. In this case it can be readily shown that the PCNN system described by (26) evolves to a solution given by the average value of the input image.

Q.E.D.

Remark: Proposition 2 shows that the PCNN model exhibits an infinite set of equilibrium points satisfying conditions $(a)$ and $(b)$, in addition to the set of equilibria (corresponding to the average value of the input image) that also the discretized Perona-Malik model possesses. As a result the two models are not topologically equivalent. The consequence of this different dynamic behavior is that the output image of a PCNN model is obtained without stopping the system evolution after a given time (scale). This could represent a significant advantage for implementation of image preprocessing algorithms, as shown in the following example.

Example: Without losing any generality we assume that a Gaussian noise with zero mean value and unknown variance is added to a given standard image (see Fig. 2). Similar considerations hold by considering different noise models (e.g. speckle or salt and pepper noise).

The upper and the lower part of Fig. 3 show the dynamic behavior of the Perona-Malik (5) and of the polynomial CNN model (10) respectively . In particular, a second order approximation of the exponential diffusivity function is exploited (see equation (4)). The parameter $m$ is set to 0.04 and the input graylevel image (bounded function between $[-1,+1]$, where -1 and +1 correspond to white and black pixels respectively) with Gaussian noise, is given as initial condition. It is readily verified that the steady-state output of the Perona-Malik model is the average value of the input image, while the PCNN converges to an equilibrium point, corresponding to an image, where the noise has been removed.

It is worth remarking that in case of model (5), the correct scale at which the evolution should be stopped, cannot be accurately estimated because no a-priori information is available for the Gaussian noise variance.

In order to compare the two processes it is useful to compute the classical signal to noise ratio defined as:

$$
S N R(t)=10 \log \frac{\sum_{i, j}\left(u_{i, j}(t)\right)^{2}}{\sum_{i, j}\left(u_{i, j}^{i d}(0)-u_{i, j}(t)\right)^{2}}
$$

where $u^{i d}(0)$ is the original input image without noise and $u_{i, j}(t)$ is the processed image. It is well known that this parameter can be used when the original image is available and the elaboration is performed on 


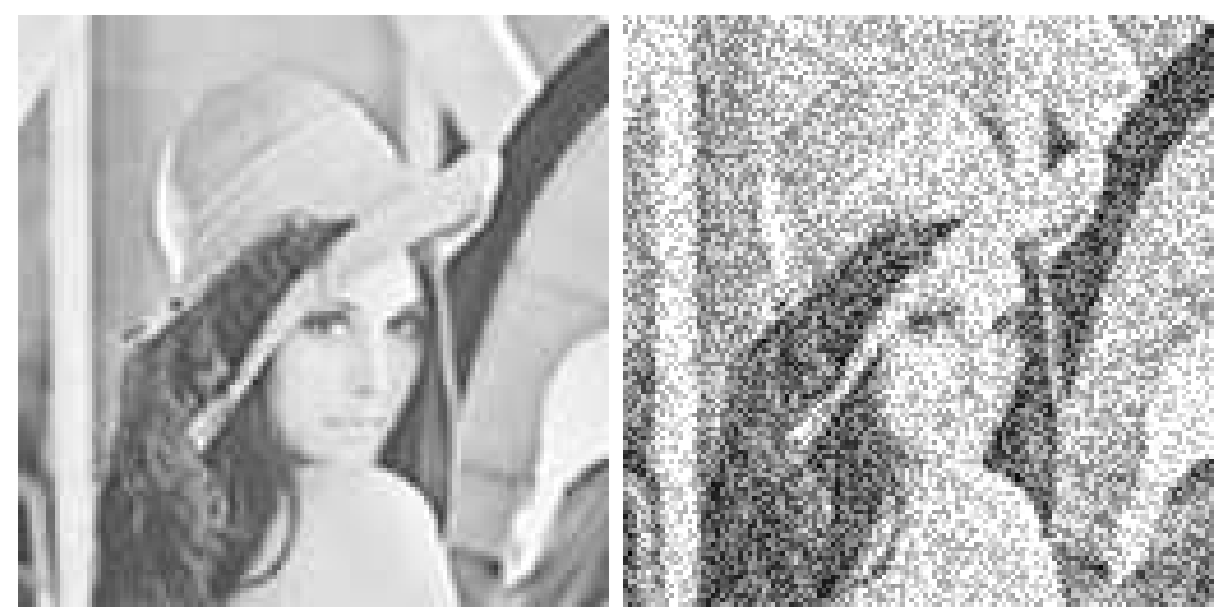

Fig. 2. Left: Original Image, Right: Image with Noise.
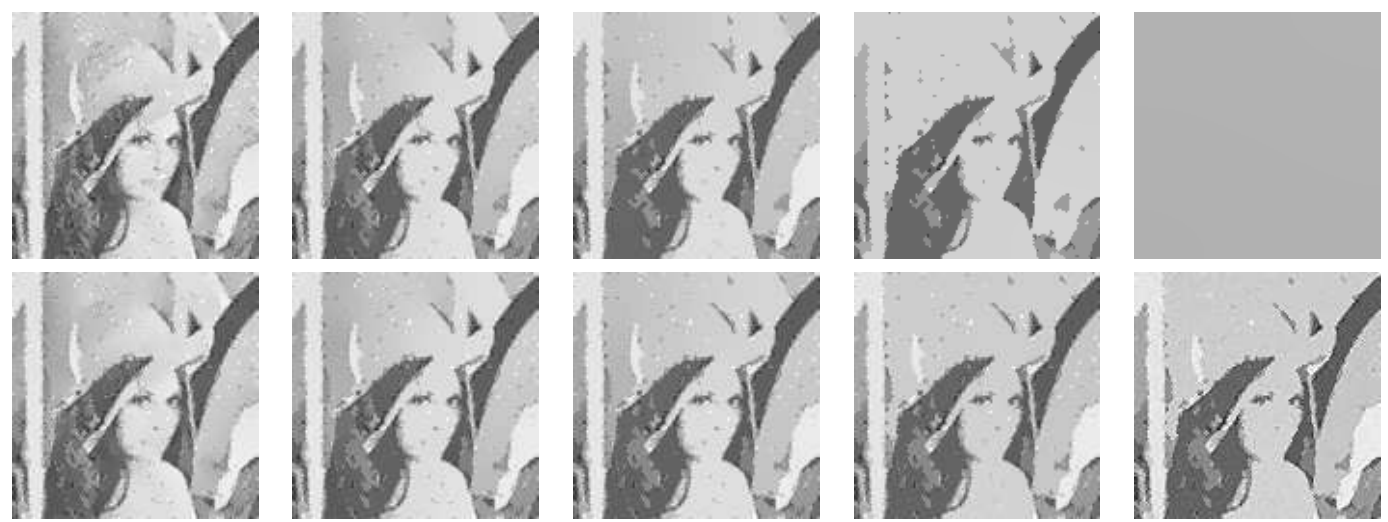

Fig. 3. Top: Perona-Malik model. Bottom: Polynomial CNN. From left to right: Output images at the scales $t=0.1, t=1, t=10$, $t=100$ and $t=500$.

static images. Table I presents the SNR values evaluated at the scales corresponding to the images shown in Fig. 3.

It is worth observing that the SNR, obtained by using the Perona-Malik model, becomes very low for large values of the scale because the output image corresponds to the average value of the noisy input image. On the other hand, by considering the PCNN model, the SNR tends to an higher constant value for the reason that the output image is one equilibrium point of the system.

\section{NON-PDE BASED CNN MODELS}

The CNN models proposed in Section II are derived from nonlinear PDE based filtering processes. Recently a novel non-PDE based approach has been proposed (see [12] and [15]), in order to deal with images corrupted by non-Gaussian noise.

This model is described by the following equation (see [12]):

\begin{tabular}{|c|c|c|c|c|c|}
\hline SNR & $t=0.1$ & $t=1$ & $t=10$ & $t=100$ & $t=500$ \\
\hline Perona-Malik model & 23.73 & 23.27 & 22.07 & 19.31 & 11.32 \\
PCNN & 23.81 & 23.00 & 22.15 & 20.70 & 19.35 \\
\hline
\end{tabular}

TABLE I

Signal to Noise Ratio evaluated at the scales $t=0.1, t=1, t=10, t=100$ and $t=500$ For the Perona-Malik AND the PCNN MODELS. 


$$
\begin{aligned}
\frac{d u_{i j}(t)}{d t}= & J_{i, j-1}\left(u_{i, j-1}-u_{i, j}\right)+J_{i, j+1}\left(u_{i, j+1}-u_{i, j}\right)+ \\
& J_{i-1, j}\left(u_{i-1, j}-u_{i, j}\right)+J_{i+1, j}\left(u_{i+1, j}-u_{i, j}\right)
\end{aligned}
$$

where the coefficients $J_{i, j-1}, J_{i, j+1}, J_{i-1, j}$, and $J_{i+1, j}$ represent the local variances defined as:

$$
\begin{aligned}
& J_{i, j-1}=\left[\sum_{l=j+1,|k-i| \leq 1}\left(u_{k, l}-u_{i, j}\right)^{2}\right] \frac{\left(1-e^{-\frac{V_{i j}}{\lambda}}\right)}{V_{t}}+0.25 e^{-\frac{V_{t}}{\lambda}} \\
& J_{i, j+1}=\left[\sum_{l=j-1,|k-i| \leq 1}\left(u_{k, l}-u_{i, j}\right)^{2}\right] \frac{\left(1-e^{-\frac{V_{i j}}{\lambda}}\right)}{V_{t}}+0.25 e^{-\frac{V_{t}}{\lambda}} \\
& J_{i-1, j}=\left[\sum_{k=i+1,|l-j| \leq 1}\left(u_{k, l}-u_{i, j}\right)^{2}\right] \frac{\left(1-e^{-\frac{V_{i j}}{\lambda}}\right)}{V_{t}}+0.25 e^{-\frac{V_{t}}{\lambda}} \\
& J_{i+1, j}=\left[\sum_{k=i-1,|l-j| \leq 1}\left(u_{k, l}-u_{i, j}\right)^{2}\right] \frac{\left(1-e^{-\frac{V_{i j}}{\lambda}}\right)}{V_{t}}+0.25 e^{-\frac{V_{t}}{\lambda}}
\end{aligned}
$$

and $V_{i j}=J_{i, j-1}+J_{i, j+1}+J_{i-1, j}+J_{i+1, j}$.

As reported in [12], the model (30) is not very sensitive to the parameter $\lambda$ for a large range of values. It is shown in [12] that piecewise linear signals are better reconstructed by non-PDE based models in presence of heavy noise corruption. The implementation of (30) onto the CNN Universal Chip (see [12]) requires space-variant linear template programming, in order to evaluate the coefficients $J_{i, j-1}, J_{i, j+1}$, $J_{i-1, j}$, and $J_{i+1, j}$.

It is easily seen that equation (30) can be approximated through the polynomial CNN model (10), by only exploiting space invariant templates.

In fact, the coefficients $J_{i, j-1}, J_{i, j+1}, J_{i-1, j}$, and $J_{i+1, j}$ can be estimated as:

$$
\begin{aligned}
& J_{i, j-1}=\left[\sum_{l=j+1,|k-i| \leq 1}\left(u_{k, l}-u_{i, j}\right)^{2}\right] \gamma_{1}\left(V_{i j}\right)+0.25 \gamma_{2}\left(V_{i j}\right) \\
& J_{i, j+1}=\left[\sum_{l=j-1,|k-i| \leq 1}\left(u_{k, l}-u_{i, j}\right)^{2}\right] \gamma_{1}\left(V_{i j}\right)+0.25 \gamma_{2}\left(V_{i j}\right) \\
& J_{i-1, j}=\left[\sum_{k=i+1,|l-j| \leq 1}\left(u_{k, l}-u_{i, j}\right)^{2}\right] \gamma_{1}\left(V_{i j}\right)+0.25 \gamma_{2}\left(V_{i j}\right) \\
& J_{i+1, j}=\left[\sum_{k=i-1,|l-j| \leq 1}\left(u_{k, l}-u_{i, j}\right)^{2}\right] \gamma_{1}\left(V_{i j}\right)+0.25 \gamma_{2}\left(V_{i j}\right)
\end{aligned}
$$

where $\gamma_{1}(\cdot)$ and $\gamma_{2}(\cdot)$ are given by equation (8) and $V_{i j}$ has the following expression:

$$
V_{i j} \cong\left[\sum_{|k-i| \leq 1,|l-j| \leq 1}\left(u_{k, l}-u_{i, j}\right)^{2}\right] .
$$


Finally, by posing $\gamma_{1}\left(V_{i j}\right)=\gamma_{2}\left(V_{i j}\right) / \lambda$ and $\gamma_{2}\left(V_{i j}\right)=\gamma_{i j}$, the following nonlinear template, whose structure is identical for each processing cell $c_{i j}$, is obtained:

$$
\left[\begin{array}{ccc}
0 & \Gamma_{i-1, j} & 0 \\
\Gamma_{i, j-1} & 0 & \Gamma_{i, j+1} \\
0 & \Gamma_{i+1, j} & 0
\end{array}\right]
$$

where

$$
\begin{aligned}
\Gamma_{i, j-1} & =\left\{\left[\sum_{l=j+1,|k-i| \leq 1}\left(u_{k, l}-u_{i, j}\right)^{2}\right]+0.25 \lambda\right\} \frac{\gamma_{i j}}{\lambda} \\
\Gamma_{i, j+1} & =\left\{\left[\sum_{l=j-1,|k-i| \leq 1}\left(u_{k, l}-u_{i, j}\right)^{2}\right]+0.25 \lambda\right\} \frac{\gamma_{i j}}{\lambda} \\
\Gamma_{i-1, j} & =\left\{\left[\sum_{k=i+1,|l-j| \leq 1}\left(u_{k, l}-u_{i, j}\right)^{2}\right]+0.25 \lambda\right\} \frac{\gamma_{i j}}{\lambda} \\
\Gamma_{i+1, j} & =\left\{\left[\sum_{k=i-1,|l-j| \leq 1}\left(u_{k, l}-u_{i, j}\right)^{2}\right]+0.25 \lambda\right\} \frac{\gamma_{i j}}{\lambda} .
\end{aligned}
$$

\section{CONCLUSIONS}

In this study we have proposed a class of CNN models for approximating isotropic (Perona-Malik) and anisotropic diffusion equations, based on a polynomial approximation of the diffusivity function. Such models present the advantage of defining a paradigm of polynomial CNNs, that is also useful for approximating some non-PDE based models.

The dynamic of the original Perona-Malik model and that of the polynomial CNN have been compared. It has been shown that polynomial CNNs have more than one stable equilibrium point and for each initial image, the output image corresponds to one of these equilibria. This property (implying that the PeronaMalik and the polynomial CNN model are not topologically equivalent) could be exploited for some image preprocessing tasks.

\section{ACKNOWLEDGMENT}

This work was supported in part by Ministero dell'Istruzione, dell'Università e della Ricerca, within the framework of the National Research Plan PRIN 2004 no. 2004092944_004 and the FIRB project no. RBAU01LRKJ.

\section{REFERENCES}

[1] J. Weickert, "A review of nonlinear diffusion filtering," Lecture Notes in Computer Science, Springer, Berlin, vol. 1252 , pp. 3-28, 1997.

[2] L. Alvarez, F. Guichard, P. L. Lions and J. M. Morel, "Axioms and fundamental equations of image processing," Arch. Rational Mech. Anal., vol. 123, pp. 199-257, 1993.

[3] A. P. Witkin, "Scale-space filtering," Proc. Eighth Int. Joint Conf. on Artificial Intelligence (IJCAI'83), vol. 2, pp. 1019-1022, 1983.

[4] F. Catté, P. L. Lions, J. M. Morel and T. Coll, "Image selective smoothing and egde detection by nonlinear diffusion," SIAM Journal on numerical analisys, vol. 29, pp. 182-193, 1992. 
[5] P. Perona, J. Malik, "Scale space and edge detection, using anisotropic diffusion," IEEE Trans. Pattern Anal. Mach. Intell., vol. 12, pp. 629-639, 1990.

[6] L. O. Chua and L. Yang, "Cellular neural networks: Theory,” IEEE Trans. Circuits Syst. - I, vol. 35, no. 10, pp. 1257-1272, Oct. 1988.

[7] L. O. Chua and L. Yang, "Cellular neural networks: Applications," IEEE Transactions on Circuits and Systems, vol. 35, no. 10, pp. 1273-1290, Oct. 1988.

[8] L. O. Chua and T. Roska, "The CNN paradigm," IEEE Trans. Circuits Syst. - I, vol. 40, no. 3, pp. 147-156, March 1993.

[9] L. O. Chua and T. Roska, Cellular neural networks and visual computing, Cambridge University Press, U.K., 2002

[10] L. O. Chua, T. Roska, T. Kozek, and A. Zarandy, "CNN universal chips crank up the computing power," IEEE Circuits and Devices, pp. 18-28, 1996

[11] M. Gilli, T. Roska, L. O. Chua, P. P. Civalleri, "CNN dynamics represents a broader class than PDEs," International Journal of Bifurcation and Chaos, vol. 12, no. 10, pp. 2051-2068, 2002.

[12] C. Recekzky, "CNN architectures for constrained diffusion based locally adaptative image processing," Int. J. Circuit Theory and Applicat., vol. 30, pp. 313-348, 2002.

[13] C. Recekzky, T. Roska and A. Ushida, "CNN-based difference-controlled adaptative nonlinear image fiters," Int. J. Circuit Theory and Applicat., vol. 26, pp. 375-423, 1998.

[14] J. Weickert and B. Benhamouda,"Nonlinear diffusion scale-spaces: From the continuous to the, discrete setting," in Proc. ICAOS96: Images, Wavelets and PDEs, vol.219, M.-O. Berger et al., Eds. New York: Springer, pp. 111-118, 1996.

[15] T. Shiota N. Nitzberg, "Non-linear image smoothing with edge and corner enhancement," Technical Report 90-2, Division of Applied Sciences, Harvard University, Cambridge, MA. 